\title{
Regulatory mechanisms underlying sepsis progression in patients with tumor necrosis factor- $\alpha$ genetic variations
}

\author{
YANGZHOU LIU*, NING HAN*, QINCHUAN LI and ZENGCHUN LI \\ Emergency Trauma Department, Shanghai East Hospital, Shanghai 200120, P.R. China
}

Received November 4, 2014; Accepted November 18, 2015

DOI: 10.3892/etm.2016.3308

\begin{abstract}
The present study aimed to investigate the regulatory mechanisms underlying sepsis progression in patients with tumor necrosis factor (TNF)- $\alpha$ genetic variations. The GSE5760 expression profile data, which was downloaded from the Gene Expression Omnibus database, contained 30 wild-type (WT) and 28 mutation (MUT) samples. Differentially expressed genes (DEGs) between the two types of samples were identified using the Student's t-test, and the corresponding microRNAs (miRNAs) were screened using WebGestalt software. An integrated miRNA-DEG network was constructed using the Cytoscape software, based on the interactions between the DEGs, as identified using the Search Tool for the Retrieval of Interacting Genes/Proteins database, and the correlation between miRNAs and their target genes. Furthermore, Gene Ontology and pathway enrichment analyses were conducted for the DEGs using the Database for Annotation, Visualization and Integrated Discovery and the KEGG Orthology Based Annotation System, respectively. A total of 390 DEGS between the WT and MUT samples, along with 11 -associated miRNAs, were identified. The integrated miRNA-DEG network consisted of 38 DEGs and 11 miRNAs. Within this network, COPS2 was found to be associated with transcriptional functions, while $F U S$ was found to be involved in mRNA metabolic processes. Other DEGs, including $F B X W 7$ and $C U L 3$, were enriched in the ubiquitin-mediated proteolysis pathway. In addition, miR-15 was predicted to target COPS 2 and CUL3. The results of the present study suggested that COPS2, FUS, FBXW7 and CUL3 may be associated with sepsis in patients with TNF- $\alpha$ genetic variations. In the progression of sepsis, $F B X W 7$ and CUL3 may participate in the ubiquitin-mediated proteolysis pathway, whereas COPS 2 may regulate the phosphorylation
\end{abstract}

Correspondence to: Dr Qinchuan Li or Dr Zengchun Li, Emergency Trauma Department, Shanghai East Hospital, 150 Jimo Road, Shanghai 200120, P.R. China

E-mail: qinchuanlii@163.com

E-mail: smztmp@126.com

*Contributed equally

Key words: tumor necrosis factor- $\alpha$ variation, sepsis, microarray data, ubiquitination, microRNA and ubiquitination of the FUS protein. Furthermore, COPS2 and CUL3 may be novel targets of miR-15.

\section{Introduction}

Multiple trauma, which is commonly associated with severe injuries and multiple organ failure, may lead to various complications, including sepsis and septic shock, which are major healthcare problems worldwide (1-3). There are 400,000-500,000 cases of sepsis in the United States annually (4). Antimicrobial therapy may be applied for the management of sepsis; however, the mortality rate associated with sepsis has increased, and was reported to be as high as $40 \%$ in 2003 (5).

Tumor necrosis factor (TNF)- $\alpha$, a cytokine that is predominantly secreted by macrophages, has been shown to be involved in the regulation of numerous biological processes, including cell proliferation, differentiation, apoptosis, lipid metabolism and coagulation (6-8). However, the role of TNF- $\alpha$ in tumorigenesis remains unclear. This cytokine has been reported to induce tumor necrosis and apoptosis, as well as to promote tumor development (9). However, previous studies investigating the role of TNF- $\alpha$ in clinical sepsis syndrome or septic shock have reported conflicting results (10-12). TNF- $\alpha$ has been established as an effective marker in the diagnosis of neonatal sepsis (13); however, the mechanisms underlying the regulatory role of TNF- $\alpha$ in the development of sepsis syndrome remain undefined. Genetic variations have previously been implicated in the progression of numerous types of cancer $(14,15)$. In addition, the clinical outcomes of sepsis have been associated with genetic polymorphisms in the genes encoding various inflammatory cytokines (16). Menges et al (17) demonstrated that common variants of the TNF- $\alpha$ gene were associated with sepsis syndrome and mortality following severe injury. Reportedly, the common TNF- $\alpha$ gene variant carrying the TNF rs1800629 A allele is correlated with higher TNF- $\alpha$ serum concentrations and alteration of genes strongly associated with proinflammatory and apoptosis (17). Furthermore, TNF rs1800629 A is closely associated with sepsis syndrome and mortality following multiple trauma (18).

The present study re-analyzed the GSE5760 microarray data deposited in the Gene Expression Omnibus (GEO) database by Menges et al (17), in order to detect genes that were differentially expressed between patients with and without TNF- $\alpha$ genetic variations, and to identify their potential functions and pathways. Furthermore, the regulatory associations 
between differentially expressed genes (DEGs) and microRNAs (miRNAs) were analyzed in order to elucidate the regulatory mechanisms underlying sepsis in patients with TNF- $\alpha$ genetic variations, at the transcriptional and post transcriptional levels.

\section{Materials and methods}

Microarray data. The GSE5760 gene expression profile data was downloaded from the GEO database (http://www.ncbi. nlm.nih.gov/geo/). Based on the description provided by Menges et al (17), the profile data consisted of 30 wild-type (WT) peripheral blood samples from 12 injured patients without the TNF- $\alpha$ rs1800629 A variant and 28 mutation (MUT) peripheral blood samples from 10 injured patients carrying the TNF- $\alpha$ rs1800629 A variant. The technical replicate numbers for the WT samples were three replicates for 6 patients and two replicates for the other 6 patients. The technical replicate numbers for the MUT samples were three replicates for 8 patients and two replicates for 2 patients. Menges et al (17) had used the GPL4204 platform (GE Healthcare/Amersham Biosciences CodeLink UniSet Human I Bioarray; GE Healthcare, Little Chalfont, UK). The annotation information in the platform was also downloaded.

Data preprocessing and identification of DEGs. The gene expression value was calculated from raw microarray data of the probe value. In the case that multiple probes corresponded to one gene, the average value was calculated as the expression level of this gene, whereas in the case that one probe corresponded to multiple genes, the probe value was removed. Following transformation of the data by $\log _{2}$ and normalization using the median method (19), DEGs between the WT and MUT samples were identified using the Limma package in R software (20) and Student's t-test. The Benjamini-Hochberg procedure (21) was applied, in order to control the false discovery rate (FDR). The threshold criteria for the DEGs were FDR $<0.05$ and $\log _{2}$ fold change (FC)| of $>0.05$.

Selection of miRNAs targeted to DEGs. Following identification of the DEGs, WebGestal software (version 2.0; Vanderbilt University, Nashville, TN, USA; http://bioinfo.vanderbilt. edu/webgestalt/) (22) was used in order to identify miRNAs that were associated with the DEGs. A threshold of adjusted $\mathrm{P}<0.05$ was used.

Construction of an integrated regulatory network between miRNAs and their target DEGs. The DEGs were mapped using the Search Tool for the Retrieval of Interacting Genes/Proteins database (http://string-db.org/) (23), in order to identify potential protein-protein interactions between the DEGs. Interaction pairs were identified using the default parameter of a combined score of $\geq 0.6$. The integrated regulatory miRNA-DEG network was constructed and visualized using the Cytoscape software (24), based on the DEG interaction pairs and the interactions between the 11 miRNAs and their target DEGs.

Function and pathway enrichment analyses for the identified $D E G s$. The biological functions of the DEGs in the established network were investigated by Gene Ontology (GO) enrichment analysis, using the Database for Annotation, Visualization and
Integrated Discovery online software (https://david.ncifcrf. gov/) (25). $\mathrm{P}<0.05$ was considered to indicate a significantly enriched GO term. In addition, pathway enrichment analyses were conducted using the KEGG Orthology Based Annotation System (KOBAS, version 2; http://kobas.cbi.pku.edu.cn/home. do), in order to identify the pathways in which the DEGs were involved. In addition, the statistical method of cumulative hypergeometric distribution was applied and $\mathrm{P}<0.05$ was considered to indicate a significantly enriched pathway.

\section{Results}

Identification of DEGs between the WT and MUT samples, and the associated miRNAs. Based on the preset criteria of $\mathrm{FDR}<0.05$ and $\log 2 \mathrm{FCl}>0.05$, a total of 390 genes were shown to be differentially expressed between the WT and MUT samples, including 238 genes that were upregulated and 152 genes that were downregulated. Based on an established threshold for miRNA searching, 11 miRNAs, including miR-141, miR-374, miR-204, miR-23, miR-182, miR-26, miR-15, miR-30, miR-34, miR-181 and miR-130, were significantly associated with the identified DEGs, and were selected for inclusion in the integrated miRNA-DEG regulatory network (Table I).

Construction of the integrated miRNA-DEG regulatory network. A total of 36 DEG interaction pairs were identified, according to their predicted protein-protein interactions. Combined with the identified DEGs targeted by miRNAs, the integrated miRNA-DEG regulatory network was constructed. This network comprised 49 nodes and 88 edges, involving 11 miRNAs and 38 DEGs, including 19 upregulated and 19 downregulated genes (Fig. 1).

Biological function and pathway annotation of the DEGs. In order to investigate the functions of the DEGs associated with TNF- $\alpha$ genetic variations, the identified DEGs were subjected to GO analysis. As presented in Table II, the DEGs were predominantly enriched in seven GO terms associated with transcriptional events, phosphorylation and RNA functions. DEGs associated with transcriptional regulation were as follows: COPS2, THRB, SFPQ, SALL1, PNRC2, CTNND2, PIM1, SF1, MAP3K10, TGFBR3, HOXA9 and WHSC1. DEGs associated with transcription were as follows: COPS2, PAPOLA, THRB, SFPQ, SALL1, PNRC2, CTNND2, SF1, HOXA9 and WHSC1. In addition, DEGs associated with GO terms such as phosphorylation and protein amino acid phosphorylation were $N T R K 2$, PIM1, MAP3K10, TGFBR3, BRD4 and PRKG1.The six DEGs associated with RNA processing were FUS, DDX17, PAPOLA, $S F P Q, S F 1$ and CPSF6. Those involved in mRNA metabolic processes, which was the most significantly enriched GO term $(\mathrm{P}=0.001632)$, included FUS, PAPOLA, SFPQ, PNRC2, SF 1 and CPSF6. DEGs, including NTRK2, PIM1, MAP3K10, TGFBR3, $B R D 4$ and GAP43, were significantly associated with positive regulation of molecular function.

KOBAS analysis was also conducted in order to identify significantly enriched pathways. Two significantly enriched pathways were identified, including the glycosphingolipid biosynthesis (including B3GNT5 and B3GALT2) and ubiquitin-mediated proteolysis, which was the most significantly enriched pathway with three DEGs (including CUL3, 
Table I. miRNAs associated with DEGs.

\begin{tabular}{|c|c|c|c|c|}
\hline miRNA & ID & DEG counts & Raw P-value & Adjusted P-value \\
\hline Has_CAGTGTT, miR-141 & DB_ID:690 & 15 & $7.29 \times 10^{-8}$ & $1.39 \times 10^{-6}$ \\
\hline Has_TATTATA, miR-374 & DB_ID:727 & 14 & $1.72 \times 10^{-7}$ & $1.63 \times 10^{-6}$ \\
\hline Has_AAAGGGA, miR-204 & DB_ID:682 & 12 & $4.49 \times 10^{-7}$ & $2.84 \times 10^{-6}$ \\
\hline Has_AATGTGA, miR-23 & DB_ID:683 & 16 & $6.71 \times 10^{-7}$ & $3.19 \times 10^{-6}$ \\
\hline Has_TTGCCAA, miR-182 & DB_ID:757 & 13 & $4.62 \times 10^{-6}$ & $1.76 \times 10^{-5}$ \\
\hline Has_TACTTGA, miR-26 & DB_ID:687 & 12 & $9.99 \times 10^{-6}$ & $3.16 \times 10^{-5}$ \\
\hline Has_TGCTGCT, miR-15 & DB_ID:666 & 17 & $1.48 \times 10^{-5}$ & $4.02 \times 10^{-5}$ \\
\hline Has_TGTTTAC, miR-30 & DB_ID:667 & 16 & $3.52 \times 10^{-5}$ & $7.43 \times 10^{-5}$ \\
\hline Has_CACTGCC, miR-34 & DB_ID:673 & 10 & 0.0001 & 0.0002 \\
\hline Has_TGAATGT,miR-181 & DB_ID:669 & 13 & 0.0003 & 0.0005 \\
\hline Has_TTGCACT, miR-130 & DB_ID:676 & 11 & 0.0006 & 0.0009 \\
\hline
\end{tabular}

miR/miRNA, microRNA; DEG, differentially expressed gene.

Table II. GO enrichment analysis of the DEGs in the integrated regulatory network.

\begin{tabular}{|c|c|c|}
\hline Term & DEGs & P-value \\
\hline GO:0016071 - mRNA metabolic process & FUS,PAPOLA, SFPQ, PNRC2, SF1, CPSF6 & 0.001632 \\
\hline GO:0006396 - RNA processing & FUS, DDX17, PAPOLA, SFPQ, SF1, CPSF6 & 0.008649 \\
\hline GO:0044093 - positive regulation of molecular function & NTRK2, PIM1, MAP3K10, TGFBR3, BRD4, GAP43 & 0.011448 \\
\hline GO:0006468 - protein amino acid phosphorylation & NTRK2, PIM1, MAP3K10, TGFBR3, BRD4, PRKG1 & 0.019140 \\
\hline GO:0045449 - regulation of transcription & $\begin{array}{l}\text { COPS2, THRB, SFPQ, SALL1, PNRC2, CTNND2, } \\
\text { PIM1, SF1, MAP3K10, TGFBR3, HOXA9, WHSC1 }\end{array}$ & 0.031480 \\
\hline GO:0016310 - phosphorylation & NTRK2, PIM1, MAP3K10, TGFBR3, BRD4, PRKG1 & 0.038131 \\
\hline GO:0006350 - transcription & $\begin{array}{l}\text { COPS2, PAPOLA, THRB, SFPQ, SALL1, PNRC2, } \\
\text { CTNND2, SF1, HOXA9, WHSC1 }\end{array}$ & 0.049845 \\
\hline
\end{tabular}

GO, gene ontology; DEG, differentially expressed gene.

Table III. Enriched pathways of the differentially expressed genes in the integrated regulatory network.

\begin{tabular}{llll}
\hline ID & Pathway & P-value & Genes \\
\hline hsa04120 & Ubiquitin mediated proteolysis & 0.02628 & CUL3, FBXW7, KLHL13 \\
hsa00601 & Glycosphingolipid biosynthesis & 0.04213 & B3GNT5, B3GALT2 \\
\hline
\end{tabular}

FBXW7 and KLHL13) (Table III). Based on the information from the integrated regulatory network (Fig. 1), both COPS2 and CUL3 were found to be targets of miR-15.

\section{Discussion}

The present study identified 390 genes that were differentially expressed between the WT and MUT (carrying TNF- $\alpha$ rs1800629 A variant) samples, and established a regulatory network containing 38 DEGs and 11 miRNAs. This network suggested that COPS 2 was predominantly associated with transcriptional functions, whereas FUS was primarily involved in mRNA metabolic processes. Other DEGs, including
FBXW7 and CUL3, were enriched in the ubiquitin-mediated proteolysis pathway. Furthermore, miR-15 was predicted to target both COPS2 and CUL3.

COPS2, also known as COP9 signalosome subunit 2 or CSN2, is an essential component of the COP9 signalosome complex, which is involved in diverse cellular processes and acts as a critical regulator of the ubiquitin conjugation pathway (26). In a previous study it was demonstrated that TNF- $\alpha$ was able to promote the stabilization of Snail (the most important transcriptional repressor of E-cadherin) and $\beta$-catenin, by inhibiting GSK-3 $\beta$-mediated phosphorylation of these proteins via the nuclear factor $-\kappa B$ and Akt signalling pathways, which in turn promoted tumor cell invasion. 


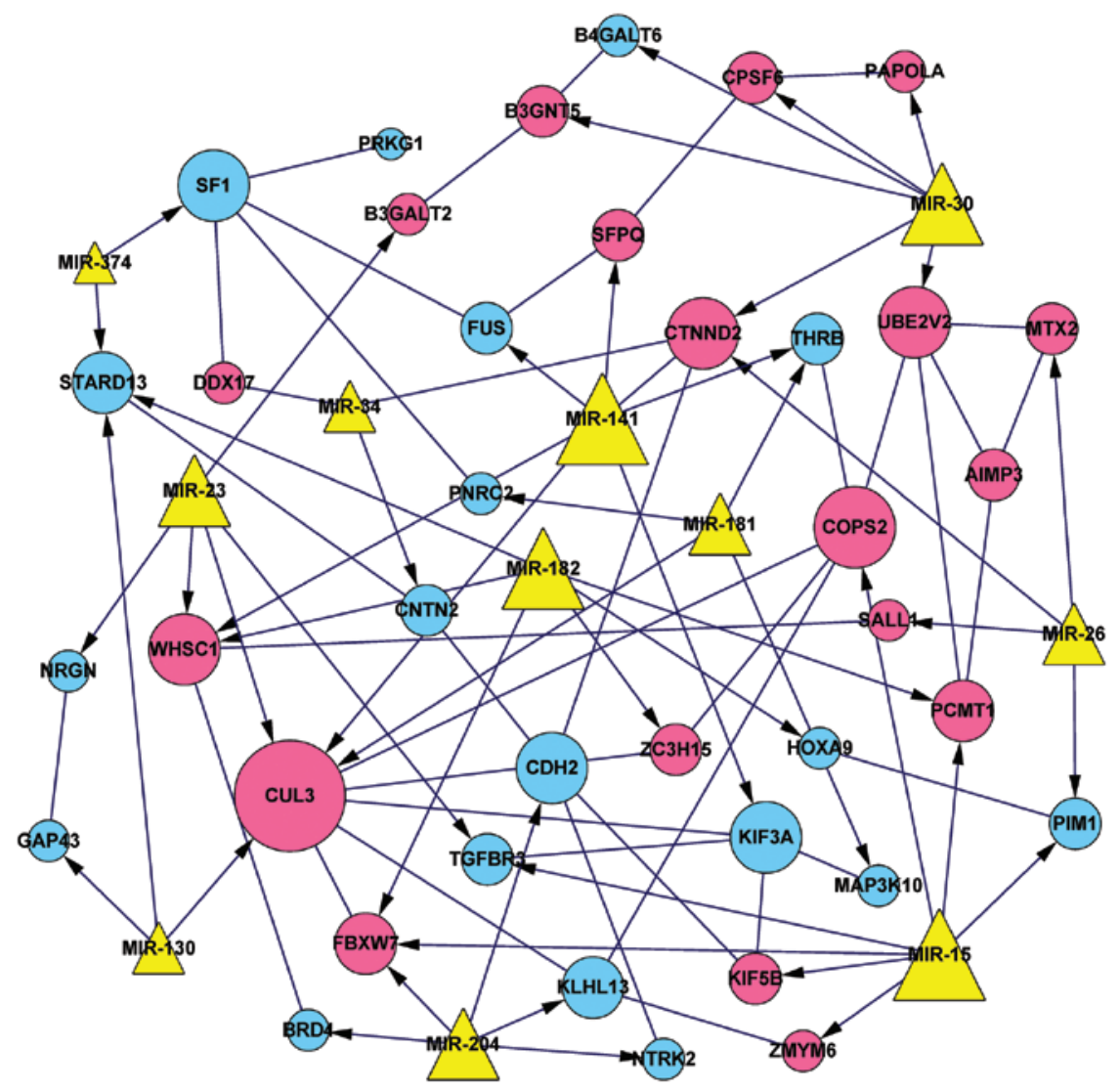

Figure 1. Integrated microRNA (miRNA)-differentially expressed gene (DEG) network. Circular nodes represent DEGs (pink circles, upregulated genes; blue circles, downregulated genes). Yellow triangles represent the miRNAs, with edges with arrowheads depicting associations between miRNAs and DEGs, and edges with lines depicting protein-protein interactions between the DEGs.

Notably, COPS2 had a crucial role in this process since it was able to inhibit the phosphorylation and ubiquitination of Snail by preventing the binding of Snail to its receptors (27).

The protein encoded by FUS is a multifunctional component of the heterogeneous nuclear ribonucleoprotein complex, which is involved in pre-mRNA splicing and the export of fully processed mRNA to the cytoplasm (28). FUS, which contains multiple RNA binding domains and an N-terminal glutamine-rich domain, acts as a mediator of RNA binding and as a potent transcriptional activator (29). In addition, FUS has previously been associated with familial amyotrophic lateral sclerosis (30) and the pathogenesis of myxoid liposarcoma (31); however, to the best of our knowledge, no previous studies have investigated the association between FUS and sepsis. In the present study, COPS2 was shown to be upregulated in the MUT samples, and was strongly associated with transcriptional functions, whereas FUS was downregulated in the TNF- $\alpha$ rs1800629 A allele variant samples, and was associated with mRNA metabolic processes. Furthermore, COPS2 and FUS were shown to interact in the integrated regulatory network. These results suggested that COPS 2 may play a vital role in the pathogenesis of sepsis in patients with TNF- $\alpha$ rs1800629 A allele variation by regulating the phosphorylation and ubiquitination of the protein encoded by $F U S$ at the transcriptional level.

FBXW7 and CUL3 are two critical DEGs, which were found to be upregulated in the TNF- $\alpha$ rs1800629 A allele variant samples. CUL3 (also known as cullin 3) encodes a member of the cullin protein family, and is the core component and scaffold protein of an E3 ubiquitin-protein ligase complex, which mediates the ubiquitiation and subsequent proteasomal degradation of target proteins (32). As major subunits of the cullin-RING ligase (CRL) family, cullins are active components of $\sim 50 \%$ of human E3 ubiquitin ligases and are responsible for one fifth of all cellular proteasome-dependent protein degradation (33). FBXW7 (also known as F-box and WD repeat domain-containing 7) is a substrate receptor for CRL1, and facilitates the ubiquitination and degradation of numerous proteins (34). Cyclin E, a member of the cyclin family, is a positive regulator of proliferation in mammalian fibroblasts. $C U L 1$ and $C U L 3$ have previously been implicated in the degradation of cyclin $E$ via two distinct pathways (35). In comparison with the CUL1/FBXW7-based E3 ligase-dependent pathway, cyclin $\mathrm{E}$ does not require phosphorylation at threonine 380, and may be recognized as a substrate by CUL3 in the CUL3-based E3 ligase pathway (36), which may be essential for the maintenance of quiescence in mammalian cells (35). Therefore, consistent with the findings of the present study, previous reports have suggested that $F B X W 7$ and $C U L 3$ may be involved in the ubiquitin-mediated proteolysis pathway $(37,38)$.

Based on a novel mechanism of autophagy, CaMKIV (also known as calcium/calmodulin-dependent protein kinase IV) may inhibit ubiquitin-mediated mTOR degradation through the inhibition of $F B X W 7$ recruitment. However, in sepsis, mTOR expression is required for autophagy (39), indicating that $F B X W 7$ may regulate mTOR. Although Qiao et al (40) did not identify $C U L 3$ as a DEG in sepsis, it is associated with DEGs 
in the PPI network, suggesting their potential involvement in sepsis. However, no study has previously reported a direct interaction between $F B X W 7$ and $C U L 3$. The aforementioned findings collectively suggest that $F B X W 7$ and $C U L 3$ may exert proteolytic functions via ubiquitination in sepsis progression in patients with TNF- $\alpha$ genetic variations. In addition, there may be regulators between $F B X W 7$ and $C U L 3$ that facilitate the functions of the two genes.

miRNAs have a critical role in the regulation of gene expression at the post-transcriptional level (41). miR-15 has been demonstrated to be associated with apoptosis in various types of cancer. For instance, a previous study identified that miR-15 was closely associated with apoptosis in chronic lymphocytic leukemia, and it was demonstrated to induce apoptosis by negatively regulating the expression of B-cell lymphoma 2 (42). Furthermore, miR-15(a/b) was reported to have inhibited the expression of cyclin E and was identified as a novel transcriptional target of E2F1, which is a vital transcription factor that induces proliferation and cell death (43). Thus, miR-15 and CUL3 may co-regulate the expression of cyclin E. Up-regulation of miR-15 has been found in the serum of neonatal sepsis patients, and this miRNA is proposed as a potential biomarker for neonatal sepsis prognosis (44). Notably, the present study identified CUL3 as a potential target of miR-15, which is consistent with this hypothesis. In addition, although previous investigations have been unable to uncover a regulatory association between miR-15 and COPS2, the present study predicted that COPS2 was a target of miR-15, thus suggesting that COPS2 may be a novel target gene for miR-15 in the progression of sepsis.

In conclusion, in the present study, COPS2, FUS, FBXW7 and $C U L 3$ were found to be associated with the progression of sepsis in patients with the TNF- $\alpha$ rs1800629 A variant. Of these genes, $F B X W 7$ and $C U L 3$ may exert regulatory roles in the ubiquitin-mediated proteolysis pathway, whereas COPS2 may affect the development of sepsis by regulating the phosphorylation and ubiquitination of the FUS protein. In addition, COPS2 and CUL3 may be novel targets of miR-15. The aforementioned genes and miRNAs may be used as therapeutic biomarkers for sepsis diagnosis in patients with the TNF- $\alpha$ rs1800629 A variant.

\section{Acknowledgements}

The present study was supported by the Shanghai Medical Key Subject Construction Project (grant no. ZK2012A28), the Shanghai Special Project for Advanced and Suitable Technology (grant no. 2013SY039) and the National Clinical Key Specialty Construction Project.

\section{References}

1. Wafaisade A, Lefering R, Bouillon B, Sakka SG, Thamm OC, Paffrath T, Neugebauer E and Maegele M; Trauma Registry of the German Society for Trauma Surgery: Epidemiology and risk factors of sepsis after multiple trauma: An analysis of 29,829 patients from the Trauma Registry of the German Society for Trauma Surgery. Crit Care Med 39: 621-628, 2011.

2. Coimbra R: From the trauma surgeon's viewpoint: Multiple injuries - which cavity to open first? J Trauma Nurs 12: 7-9, 2005.

3. Dellinger RP, Levy MM, Carlet JM, Bion J, Parker MM, Jaeschke R, Reinhart K, Angus DC, Brun-Buisson C, Beale R, et al: Surviving Sepsis Campaign: International guidelines for management of severe sepsis and septic shock: 2008. Intensive Care Med 34: 17-60, 2008.
4. Balk RA: Severe sepsis and septic shock: Definitions, epidemiology, and clinical manifestations. Crit Care Clin 16: 179-192, 2000.

5. Hanna NF: Sepsis and septic shock. Adv Emerg Nurs J 25: 158-165, 2003.

6. Widera D, Mikenberg I, Elvers M,Kaltschmidt C and Kaltschmidt B: Tumor necrosis factor $\alpha$ triggers proliferation of adult neural stem cells via IKK/NF-kappaB signaling. BMC Neurosci 7: 64, 2006.

7. Lemaittre N, Sebbane F, Long D and Hinnebusch BJ: Yersinia pestis YopJ suppresses tumor necrosis factor alpha induction and contributes to apoptosis of immune cells in the lymph node but is not required for virulence in a rat model of bubonic plague. Infect Immun 74: 5126-5131, 2006.

8. Greenberg JD, Kremer JM, Curtis JR, Hochberg MC, Reed G, Tsao P, Farkouh ME, Nasir A, Setoguchi S and Solomon DH; CORRONA Investigators: Tumour necrosis factor antagonist use and associated risk reduction of cardiovascular events among patients with rheumatoid arthritis. Ann Rheum Dis 70: 576-582, 2011.

9. Yu M, Zhou X, Niu L, Lin G, Huang J, Zhou W, Gan H, Wang J, Jiang X, Yin B and Li Z: Targeting transmembrane TNF- $\alpha$ suppresses breast cancer growth. Cancer Res 73: 4061-4074, 2013.

10. Liang Y, Li X, Zhang X, Li Z, Wang L, Sun Y, Liu Z and Ma X: Elevated levels of plasma TNF- $\alpha$ are associated with microvascular endothelial dysfunction in patients with sepsis through activating the NF- $\kappa \mathrm{B}$ and p38 mitogen-activated protein kinase in endothelial cells. Shock 41: 275-281, 2014.

11. Rice TW and Bernard GR: Therapeutic intervention and targets for sepsis. Annu Rev Med 56: 225-248, 2005.

12. Alexander JJ, Jacob A, Cunningham P, Hensley L and Quigg RJ: TNF is a key mediator of septic encephalopathy acting through its receptor, TNF receptor-1. Neurochem Int 52: 447-456, 2008.

13. Kocabas E, Sarikçioğlu A, Aksaray N, Seydaoğlu G, Seyhun Y, and Yaman A: Role of procalcitonin, C-reactive protein, interleukin-6, interleukin-8 and tumor necrosis factor-alpha in the diagnosis of neonatal sepsis. Turk J Pediatr 49: 7-20, 2007.

14. Shigematsu H, Lin L, Takahashi T, Nomura M, Suzuki M, Wistuba II, Fong KM, Lee H, Toyooka S, Shimizu N, et al: Clinical and biological features associated with epidermal growth factor receptor gene mutations in lung cancers. J Natl Cancer Inst 97: 339-346, 2005.

15. Lièvre A, Bachet J-B, Le Corre D, Boige V, Landi B, Emile JF, Côté JF, Tomasic G, Penna C, Ducreux M, et al: KRAS mutation status is predictive of response to cetuximab therapy in colorectal cancer. Cancer Res 66: 3992-3995, 2006.

16. Sutherland AM and Walley KR: Bench-to-bedside review: Association of genetic variation with sepsis. Crit Care 13: 210, 2009.

17. Menges T, König IR, Hossain H, Little S, Tchatalbachev S, Thierer F, Hackstein H, Franjkovic I, Colaris T, Martens F, et al: Sepsis syndrome and death in trauma patients are associated with variation in the gene encoding tumor necrosis factor. Crit Care Med 36: 1456-1462, e1-e6, 2008.

18. Chen G, Han N, Li G, Li X, Li G, Liu Y, Wu W, Wang Y, Chen Y, Sun G et al: Prediction of feature genes in trauma patients with the TNF rs1800629 A allele using support vector machine. Comput Biol Med 64: 24-29, 2015.

19. Fujita A, Sato JR, Rodrigues LO, Ferreira CE, and Sogayar MC: Evaluating different methods of microarray data normalization. BMC Bioinformatics 7: 469, 2006.

20. Smyth GK: Limma: Linear models for microarray data. In: Bioinformatics and computational biology solutions using $\mathrm{R}$ and Bioconductor. Gentleman R, Carey VJ, Huber W, Irizarry RA and Dudoit S (eds). Springer, New York, pp. 397-420, 2005.

21. Benjamini Y and Hochberg Y: Controlling the false discovery rate: A practical and powerful approach to multiple testing. J R Stat Soc Series B Stat Methodol 57: 289-300, 1995.

22. Duncan D, Prodduturi N and Zhang B: WebGestalt2: An updated and expanded version of the Web-based Gene Set Analysis Toolkit. BMC Bioinformatics 11 (Suppl 4): 10, 2010.

23. Franceschini A, Szklarczyk D, Frankild S, Kuhn M, Simonovic M, Roth A, Lin J, Minguez P, Bork P, von Mering C and Jensen LJ: STRING v9.1: Protein-protein interaction networks, with increased coverage and integration. Nucleic Acids Res 41: D808-D815, 2013.

24. Smoot ME, Ono K, Ruscheinski J, Wang P-L and Ideker T: Cytoscape 2.8: New features for data integration and network visualization. Bioinformatics 27: 431-432, 2011.

25. Huang W, Sherman BT and Lempicki RA: Systematic and integrative analysis of large gene lists using DAVID bioinformatics resources. Nat Protoc 4: 44-57, 2009. 
26. Wei N and Deng XW: The COP9 signalosome. Annu Rev Cell Dev Biol 19: 261-286, 2003.

27. Wu Y and Zhou BP: TNF-alpha/NF-kappaB/Snail pathway in cancer cell migration and invasion. Br J Cancer 102: 639-644, 2010.

28. Schwartz JC, Podell ER, Han SS, Berry JD, Eggan KC and Cech TR: FUS is sequestered in nuclear aggregates in ALS patient fibroblasts. Mol Biol Cell 25: 2571-2578, 2014.

29. Bentmann E, Neumann M, Tahirovic S, Rodde R, Dormann D and Haass C: Requirements for stress granule recruitment of fused in sarcoma (FUS) and TAR DNA-binding protein of 43 kDa (TDP-43). J Biol Chem 287: 23079-23094, 2012.

30. Maruyama $H$, Morino $H$, Ito $H$, Izumi $Y$, Kato $H$, Watanabe $Y$, Kinoshita Y, Kamada M, Nodera H, et al: Mutations of optineurin in amyotrophic lateral sclerosis. Nature 465: 223-226, 2010.

31. Riggi N, Cironi L, Provero P, Suvà ML, Stehle JC, Baumer K, Guillou L and Stamenkovic I: Expression of the FUS-CHOP fusion protein in primary mesenchymal progenitor cells gives rise to a model of myxoid liposarcoma. Cancer Res 66: 7016-7023, 2006.

32. Pintard L, Willis JH, Willems A, Johnson JL, Srayko M, Kurz T, Glaser S, Mains PE, Tyers M, Bowerman B and Peter M: The BTB protein MEL-26 is a substrate-specific adaptor of the CUL-3 ubiquitin-ligase. Nature 425: 311-316, 2003.

33. Saito N, Sakakibara K, Sato T, Friedman JM, Kufe DW, VonHoff DD and Kawabe T: CBS9106-induced CRM1 degradation is mediated by cullin ring ligase activity and the neddylation pathway. Mol Cancer Ther 13: 3013-3023, 2014.

34. Tron AE, Arai T, Duda DM, Kuwabara H, Olszewski JL, Fujiwara Y, Bahamon BN, Signoretti S, Schulman BA and DeCaprio JA: The glomuvenous malformation protein Glomulin binds Rbx1 and regulates cullin RING ligase-mediated turnover of Fbw7. Mol Cell 46: 67-78, 2012.

35. McEvoy JD, Kossatz U, Malek N and Singer JD: Constitutive turnover of cyclin $\mathrm{E}$ by $\mathrm{Cul} 3$ maintains quiescence. Mol Cell Biol 27: 3651-3666, 2007
36. Singer JD, Gurian-West M, Clurman B and Roberts JM: Cullin-3 targets cyclin $\mathrm{E}$ for ubiquitination and controls $\mathrm{S}$ phase in mammalian cells. Genes Dev 13: 2375-2387, 1999.

37. Eide PW, Cekaite L, Danielsen SA, Eilertsen IA, Kjenseth A, Fykerud TA, Ågesen TH, Bruun J, Rivedal E, Lothe RA and Leithe E: NEDD4 is overexpressed in colorectal cancer and promotes colonic cell growth independently of the PI3K/PTEN/AKT pathway. Cell Signal 25: 12-18, 2013.

38. Yu S, Yi H, Wang Z and Dong J: Screening key genes associated with congenital heart defects in Down syndrome based on differential expression network. Int J Clin Exp Pathol 8: 8385-8393, 2015.

39. Zhang X, Howell GM, Guo L, Collage RD, Loughran PA, Zuckerbraun BS and Rosengart MR: CaMKIV-dependent preservation of mTOR expression is required for autophagy during lipopolysaccharide-induced inflammation and acute kidney injury. J Immunol 193: 2405-2415, 2014.

40. Qiao FS, Wei C, Yun J and Qian LX: Insights into the molecular mechanisms in sepsis with microarray technology. Eur Rev Med Pharmacol Sci 18: 2405-2412, 2014.

41. Geng J, Luo H, Pu Y, Zhou Z, Wu X, Xu W and Yang Z: Methylation mediated silencing of miR-23b expression and its role in glioma stem cells. Neurosci Lett 528: 185-189, 2012.

42. Cimmino A, Calin GA, Fabbri M, Iorio MV, Ferracin M, Shimizu M, Wojcik SE, Aqeilan RI, Zupo S, Dono M, et al: miR-15 and miR-16 induce apoptosis by targeting BCL2. Proc Natl Acad Sci USA 102: 13944-13949, 2005.

43. Ofir M, Hacohen D and Ginsberg D: MiR-15 and miR-16 are direct transcriptional targets of E2F1 that limit E2F-induced proliferation by targeting cyclin E. Mol Cancer Res 9: 440-447, 2011.

44. Wang X, Wang X, Liu X, Wang X, Xu J, Hou S, Zhang X and Ding Y: miR-15a/16 are upregulated in the serum of neonatal sepsis patients and inhibit the LPS-induced inflammatory pathway. Int J Clin Exp Pathol 8: 5683-5690, 2015. 